\title{
viewpoint
}

\section{Lab-scale intervention}

\author{
SSS Science \& Society Series on Convergence Research
}

\author{
Daan Schuurbiers \& Erik Fisher
}

$\mathrm{F}$ rom mobile phones and laptop computers to in vitro fertilization and social networks on the Internet, technological devices, products and services are increasingly shaping the lives of people around the world. The pervasiveness of technology and the underlying science that makes it possible has led to a certain ambivalence: most people trust that 'science' will eventually help them to live longer, healthier and happier lives. However, they also feel increasingly uncomfortable about certain new technologies, often those that challenge or improve on 'nature'. Genetically modified crops, gene therapy, stem cell research, cloning, renewed interest in nuclear power: the list of controversial topics involving science and technology is growing steadily and debates on these topics regularly occupy centre stage in public and political arenas.

\section{...the research process itself constitutes a largely overlooked opportunity for addressing social concerns}

Policy-makers have responded by calling for increased attention to be paid to the ethical, legal and social aspects of scientific research and technological developments. In particular, new and emerging areas of research-such as genomics, synthetic biology and nanotechnology-have been accompanied by studies of their broader societal implications as well as publicengagement efforts, in order to guide research and development in ways that respect societal concerns. Such attempts to shape technological trajectories have traditionally occurred both before scientific research, for example, through research policy, technology assessment or public participation, and afterwards, through regulations or market mechanisms. Although these stages are crucial points at which to intervene, the research process itself constitutes a largely overlooked opportunity for addressing social concerns.

Indeed, if one acknowledges the central role that scientific research has in the innovation process, this is an area well worth examining. Shaping technological trajectories will, at some point, include shaping the very research processes that help to characterize them (Fisher et al, 2006). Social and ethical work in the laboratory is thus a logical next step.

The 'lab-scale' idea is catching on. An independent review of the social and ethical challenges of synthetic biology, commissioned by the Biotechnology and Biological Sciences Research Council (BBSRC; Swindon, UK), recommends that, "the scientific community must take, and be seen to be taking, a lead in debating the implications of their research and engaging with broader society around the issues raised by synthetic biology. [...] Partnership with civil society groups, social scientists and ethicists should be pursued as a highly effective way of understanding critical issues, engaging with publics and winning support for emerging scientific fields" (Balmer \& Martin, 2008). Similarly, the Netherlands Genomics Initiative (NGl; The Hague, the Netherlands) aims at "[s]trengthening the integration and interaction between the social research and the genomics research" (NGI, 2005).

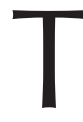
hese pronouncements seem to herald a new take on science. However, are there truly opportunities to incorporate societal concerns into the practice of science? Can this be done without compromising research integrity or hampering its productivity? Addressing these questions requires innovative ways to integrate societal research with the processes of science and technology, and new forms of collaboration among social and human scientists and natural scientists and engineers. Yet, although this type of integration makes for catchy rhetoric, one can point to few successful examples. This is partly because it is often unclear how broader considerations can be brought to bear on actual 'bench work' in ways that add value to both science and society.

\section{Shaping technological trajectories will, at some point, include shaping the very research processes that help to characterize them...}

In light of this challenge, new forms of interaction are developing between social and natural scientists to strengthen the connections between science and society. As Jacques Dubochet has observed, "Given the public's increasing interest in and scrutiny of the life sciences, it is important that natural scientists engage in debates with their colleagues from the social sciences and humanities about the implications and social impact of their work" (Dubochet, 2008). Similarly, social scientists and humanists have started to undertake 'convergence work' (Stegmaier, 2009); interdisciplinary research aimed at crossing the boundaries between natural and social sciences. In a previous issue of EMBO reports, Jane Calvert and Paul Martin discussed the possible roles of social scientists in synthetic 
biology (Calvert \& Martin, 2009). This type of 'integration' work is being conducted by the Center for Nanotechnology in Society at Arizona State University (CNS-ASU; Tempe, AZ, USA), as well as by several other laboratories and centres (Guston \& Sarewitz, 2002; Doubleday, 2007; Gorman et al, 2004; Bennett \& Rabinow, 2008).

One particular way of shaping interactions between natural and social scientists was developed by Erik Fisher at the University of Colorado at Boulder (CO, USA), with input from the engineering research group with which he had worked (Fisher, 2007). This approach, called midstream modulation, aims to construct collaborative engagement between social and natural scientists in the laboratory, in order to broaden research decisions incrementally. The term 'modulation' was introduced by Arie Rip, Professor of Philosophy of Science and Technology at the University of Twente (Enschede, the Netherlands), as an alternative to more forceful attempts to bring about change. As Rip has commented, "quasi-autonomous dynamics of science appear to be so strong that governance actors cannot do much more than try to modulate what is going on anyway" (Rip, 2006).

Fisher notes that 'midstream' denotes the phase of the research and development before scientific results are translated into products or services, but after authorization and funding decisions have been taken. It is the phase that takes place within the research laboratory, at the drawing board and wherever decisions are made about the conduct of research. Midstream modulation asks how research is to be carried out, which is the main business of research, rather than whether a research project should be carried out, which is an upstream policy question (Fig 1). It is a means to evaluate and adjust research decisions in light of societal factors while the research process is taking place.

A first proof of principle for midstream modulation was established in 2006 in the Department of Mechanical Engineering at the University of Colorado at Boulder. Fisher interacted with three graduate engineering researchers for 12 weeks as an 'embedded humanist' to identify and assess opportunities for influencing research decisions in accordance with societal concerns. He worked with researchers in the laboratory and discussed with them the decisions that they were making while

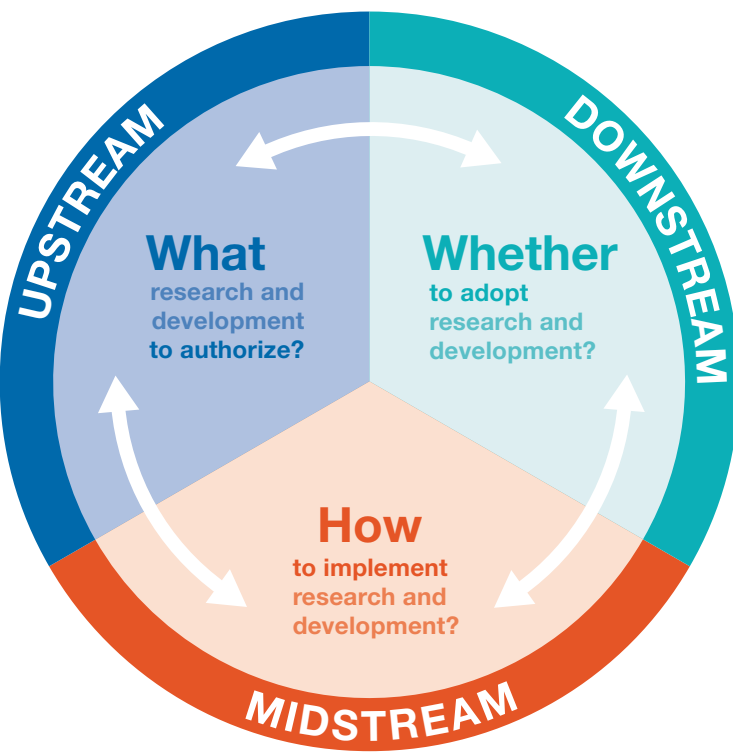

Fig 1 Stages in the governance of science and technology.

they were making them. The study focused not on the nature of societal concerns, but on the nature of engineering research decisions. The goal of the study was to assess the capacity of researchers to integrate social considerations by 'modulating' their decisions. The interaction consisted of closely following and documenting the research, attending laboratory meetings and articulating the decisions made through the use of a 'decision protocol' (Fig 2). Pre-interaction and post-interaction interviews were held with all participants to measure any significant differences in awareness or attitude. Each week, the protocol was used to discuss and explore decision opportunities, the considerations they invoked, and a list of perceived alternatives and potential outcomes (Fisher, 2007; Fisher \& Mahajan, 2006).

\section{Midstream modulation asks how research is to be carried out, which is the main business of research, rather than whether a research project should be carried out...}

This approach allowed Fisher to map numerous social, physical and cognitive 'modulators' that influence research decisions, including the participants' awareness of those modulators. Research decisions were found to be subject to subtle societal influences, and-more significantly-researchers were found to become aware of the possibility of modulating their decisions. Rather than experiencing societal considerations as 'ethical speed bumps' imposed on their projects, the participants indicated that such reflections broadened their decisions. They realized that they were making choices, that these choices were based on a range of considerations, and that by reflecting on them, they found that decision outputs and inputs can both vary.

Fisher made no attempt to alter research decisions, only to stimulate awareness of the possibility of doing so. Still, as a result of the ongoing interactions between the natural and social researchers, and in response to environmental, health and safety considerations, research practices themselves changed: an experimental setup was modified, disposal methods were altered, an alternative chemical catalyst was introduced and safety rules were formulated. This case study implies that midstream modulation can create 'dual value' by advancing both general social values such as environmental health and safety, and more specific laboratory research objectives such as generating more effective research decisions leading to publishable data (Fisher \& Mahajan, 2006).

nother iteration of midstream mod-
ulation was recently carried out at
the Department of Biotechnology
at Delft University of Technology in the 


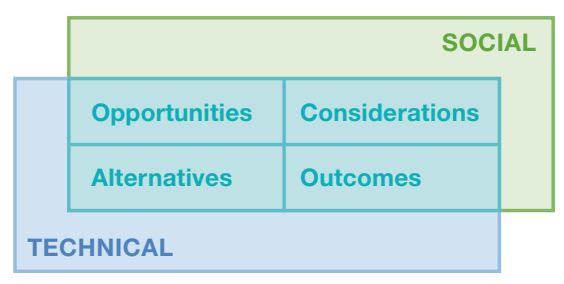

Fig 2 | The decision protocol.

Netherlands as part of a research project of the Centre for Society and Genomics (Nijmegen, the Netherlands) on social responsibility in science. The study assessed whether interdisciplinary decision-making could help to bring broader social considerations to bear on the scientific activities of researchers in the laboratory. For 12 weeks, Daan Schuurbiers interacted regularly with research participants-doing so more as a collaborator than an observer. In the process of building a shared and detailed understanding of the goals and considerations of research, Schuurbiers and his participants addressed a range of topics of mutual interest. These included environmental health and safety, sustainability, patenting, the role of private funding in academic research, long-term research strategies and the responsibility of scientists to communicate with society.

This second study corroborated the utility of midstream modulation as a tool for social responsibility in science. The participants who interacted with Schuurbiers showed increasing awareness that technological developments are shaped by political, economic and social concerns, as well as by 'purely scientific' considerations. Putting scientific research into social perspective made the researchers think about their decisions in new ways. One participant commented: "You have a different point of view -it makes me think differently about what I do." Another mentioned: "It's good to think about what your research can deliver for society." Ongoing discussions during laboratory work brought the connections between the societal implications of research decisions into perspective. The research participants came to see connections between their scientific activities and wider cultural practices (Webster, 2007). These discussions altered what one might call the researchers' 'conscience value' towards the social and ethical aspects of research: during the interactions it became clear that ethical and social considerations need not necessarily apply only to obviously ethically sensitive topics, but that it often makes sense to address broader considerations in less contested areas of science.

$\mathrm{T}$ hese two examples of lab-scale social science also provide learning opportunities for the social researchers. As engineering ethicist Ibo van de Poel, from the Centre for Ethics and Technology at Delft University of Technology, has noted, ethical questions acquire meaning within the context of research (van de Poel \& Verbeek, 2006). When such ethical issues arise 'bottom-up', their power and the perceived need to think about them become all the more apparent. Thus, embedded scholars also become more aware of the social and institutional constraints in which researchers have to operate, such as balancing scientific and commercial interests, coping with the demands of funding agencies and negotiating expectations with supervisors.

\section{Social scientists need to become sensitive to the day-to- day complexities of research practices, whereas natural scientists and engineers should be challenged to think about their research in different ways...}

The midstream-modulation approach helps to broker fruitful cross-cultural interactions that allow social and natural researchers to work on the same project, albeit from their own unique disciplinary and cultural standpoints. It provides an interactive space in which participants act as both experts and laypersons simultaneously. Yet, the extent to which midstream modulation can consistently lead to enhanced research outcomes remains an open question. Although the first study mentioned in this article documented four changes in research practices, the second has not yet produced similar outcomes. As one participant from the second study commented: "Does it change my thinking? Yes. Does it change what I do on a daily basis? No." The general utility of midstream modulation will need to be assessed based on a larger set of studies.

Midstream modulation will be put to the test in 2009 and 2010, when 20 case studies will be carried out in laboratories throughout the world, as part of the SocioTechnical Integration Research (STIR) project that Fisher is leading (http://cns.asu.edu/ stir). Funded by the US National Science Foundation (Arlington, VA, USA) and organized by the CNS-ASU, the STIR project will introduce social science and the humanities into research centres and institutes in North America, Western Europe and East Asia. Performing the research at several sites might help to clarify the basic conditions necessary for successful interdisciplinary collaborations that are aimed at responsible science and innovation. At a higher level of abstraction, the project will investigate the considerations that drive research agendasnormative ideals, technical challenges and funding opportunities - and that shape scientists' visions of the future and ultimately influence policy decisions.

I n summary, lab-scale interactions can improve the relevance of both social and natural scientific research. In applying their knowledge from the social sciences and humanities to laboratory research practices, embedded scholars must learn to adjust their expectations to what is scientifically and practically feasible. This might help to turn undergrounded 'moralism' and idealistic visions of the responsibility of scientists into more realistic and practical outcomes. Social scientists need to become sensitive to the day-to-day complexities of research practices, whereas natural scientists and engineers should be challenged to think about their research in different ways - and to acknowledge the rationality of alternative views and approaches. Ideally, the intangible concepts of sustainability, environmental justice and social equity will become more visible within scientific practice itself.

Numerous challenges remain in trying to connect science and society in the laboratory. Social and natural researchers will always have different ways of looking at the world, approaching problems and finding solutions. Colleagues and peers might greet boundary-crossing attempts with suspicion, and laboratory researchers will question the value of considering the social dimensions of their work if it does not allow for more immediate practical insights. Social researchers, by contrast, might worry about becoming co-opted, being limited to more benign forms of critique or becoming 'token ethicists' used to deflect societal concerns. Another important challenge is that the applications and consequences of research 


\section{SSS Science \& Society Series on Convergence Research}

This article is part of the EMBO reports Science \& Society Series on Convergence Research, which features Viewpoints from authors who attended the 'Doing Society and Genomics—Convergence and Competence Building' workshop organized by Peter Stegmaier for the Centre for Society and Genomics at Radboud University (Nijmegen, the Netherlands) in September 2008. We hope that this Viewpoint series will help to introduce our readers to the new multi- and transdisciplinary developments among the life sciences and the social sciences and humanities.

are notoriously unpredictable. We suspect that it might be easier to address societal concerns that involve research processes such as experimental procedures, than to tackle those that involve applications and end-user considerations.

Despite such challenges, these projects have generated enthusiastic responses from natural and social scientists alike. The emergence of cooperative schemes in research and education constitutes an exciting window of opportunity for strengthening connections between science and society. In concert with ongoing activities in technology assessment, upstream engagement and engineering ethics, midstream modulation of research processes can identify and take advantage of opportunities to influence long-term outcomes. Furthermore, it can instil the habit of reflecting on how decisions might be made with respect to broader societal concerns. Our two studies show that ethical considerations are a substantial part of scientific and technological research, and that making these considerations explicit may contribute towards more socially resilient scientific practices without jeopardizing the science. As more researchers open up to collaborative engagement on the role of science in society, notable changes in education, training and conduct may well appear. Such opportunities for reflection ought to be cultivated, but they need to respect the operative conditions of laboratory culture if they are to lead to integration that is indeed perceived to have 'dual value'.

There is never one technologically determined path to progress. It is therefore legitimate to ask how we want science and technology to serve society. As Stephen Cohen and Damian Grace comment, the 'social rationale' for science and engineering is to develop and apply scientific knowledge to the public good (Cohen \& Grace,
1994). Notwithstanding the fact that innovation is not a linear process and that we cannot accurately predict which research result will lead to which innovation, we can nevertheless work towards a system in which the principles of efficiency and objectivity are integrated with the consideration of public values and ethical concerns. Addressing the ethical, legal and social aspects of scientific practice might, in this way, change from a 'tick-the-box' exercise to a normal part of scientific reasoning.

\section{ACKNOWLEDGEMENTS}

The researchers at the Delft University of Technology Department of Biotechnology and the University of Colorado at Boulder Department of Mechanical Engineering are gratefully acknowledged for their collaboration. This article is the result of a research project of the Centre for Society and Genomics and the Kluyver Centre for Genomics of Industrial Fermentation in the Netherlands, funded by the Netherlands Genomics Initiative. This material is based upon work supported by the US National Science Foundation under grant number 0531194.

\section{REFERENCES}

Balmer A, Martin P (2008) Synthetic Biology: Social and Ethical Challenges. An Independent Review Commissioned by the Biotechnology and Biological Sciences Research Council (BBSRC). Swindon, UK: BBSRC

Bennett G, Rabinow P (2008) Human Practices 2007. Interfacing Three Modes of Collaboration. Houston, TX, USA: Rice University, the Connexions Project. http://cnx.org

Calvert J, Martin P (2009) The role of social scientists in synthetic biology. EMBO Rep 10: 194-200

Cohen S, Grace D (1994) Engineers and social responsibility: an obligation to do good. IEEE Technology and Society Magazine 13: 12-19

Doubleday R (2007) The laboratory revisited: academic science and the responsible development of nanotechnology. NanoEthics $\mathbf{1}$ : 167-176

Dubochet J (2008) Citizen biologists: the Lausanne experience. EMBO Rep 9: 5-9

Fisher $\mathrm{E}$ (2007) Ethnographic invention: probing the capacity of laboratory decisions. NanoEthics 1: 155-165

Fisher E, Mahajan R (2006) Midstream modulation of nanotechnology in an academic research laboratory. In Proceedings of IMECE2006: American Society of Mechanical Engineers International Mechanical Engineering Congress and Exposition, November 5-10. Chicago, IL, USA: ASME

Fisher E, Mitcham C, Mahajan R (2006) Midstream modulation of technology: governance from within. Bull Sci Technol Soc 26: $485-496$

Gorman ME, Groves JF, Shrager J (2004) Societal dimensions of nanotechnology as a trading zone: results from a pilot project. In Discovering at the Nanoscale, D Baird, A Nordmann, J Schummer (Eds). Amsterdam, the Netherlands: IOS

Guston DH, Sarewitz D (2002) Real-time technology assessment. Technol Soc 24: 93-109

NGI (2005) Genomics 2008-2012. Building and Utilising. The Dutch Genomics Infrastructure 2008-2012. The Hague, the Netherlands: Netherlands Genomics Initiative. www.genomics.nl

Rip A (2006) A co-evolutionary approach to reflexive governance- and its ironies. In Reflexive Governance for Sustainable Development, J Voss, D Bauknecht, R Kemp (Eds), pp 82-100. Cheltenham, UK: Edward Elgar

Stegmaier P (2009) The rock ' $\mathrm{n}$ ' roll of knowledge co-construction. An introduction to the Science \& Society series on convergence research inspired by the 'Doing Society and Genomics' Workshop, 2008. EMBO Rep 10: 114-119

van de Poel I, Verbeek PP (2006) Ethics and engineering design. Sci Technol Human Values 31: $223-236$

Webster A (2007) Crossing boundaries: social science in the policy room. Sci Technol Human Values 32: 458-478

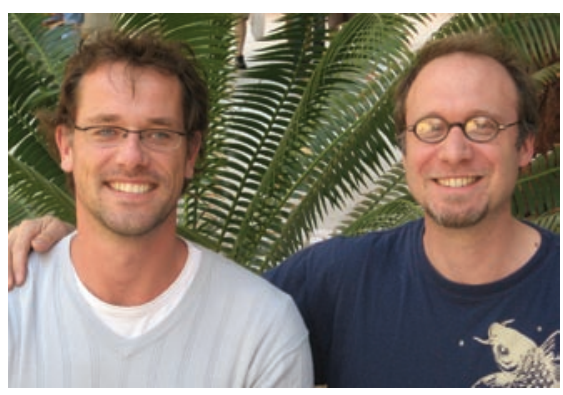

Daan Schuurbiers (left) is a researcher at the Working Group on Biotechnology and Society, Department of Biotechnology, Delft University of Technology, the Netherlands. E-mail:d.schuurbiers@tudelft.nl Erik Fisher is an assistant research professor at the Center for Nanotechnology in Society, Consortium for Science, Policy and Outcomes, Arizona State University, Arizona, USA. E-mail: efisher1@asu.edu

doi:10.1038/embor.2009.80 